\title{
Impulsive Spraying Insecticide Model with Single Species
}

\author{
B.B. Gao \\ College of science \\ Zhejiang University of technology \\ Hangzhou, China
}

\author{
D.J. Wang \\ College of science \\ Zhejiang University of technology \\ Hangzhou, China
}

\begin{abstract}
Here we discussed the SIRS model of pulse spaying pesticides with vertical transmission and single species. Assuming that the incidence rate is periodically time-dependant and the total number of forest trees also changes over time, we studied the stability of impulsive model using piecewise continuous Lyapunov function and differential theories. Then we obtained the conditions of global asymptotic stability (GAS) for the periodic solution of pests being relatively or totally eradicated. Finally, we selected appropriate data and used numerical examples to show the correctness of the conclusions.
\end{abstract}

Keywords- pulse spraying insecticide; the plants diseases and insect pests model; the disease-free periodic solution; stability; global stability

\section{INTRODUCTION}

Using chemical control is one of the regular methods for modern control of plant diseases and insect pests, that is to say, people spray insecticide for plants to achieve the goal of killing pests. Therefore, in the same way to the epidemic model of [1-2],we can also set up SIRS model of plants disease and insect pests

Many models suppose that the spraying insecticide behavior is a continuous process (see[3-6]).However, this assumption neither as cheap nor as environmentally benign [7]. Therefore, studying impulsive model has drawn more attention [8-10].

Assumpting the total number of forest plants is constant, people established many mathematical models(see[1113]).As the model can be reduced to planar system, they are easy to study and the results appear mostly intact. However if birth rate is not equal to mortality rate or it exists population migration and so on, the total number of forest plants is not constant and our models will not be reduced. As a consequence, we need to discuss our questions in threedimensional space and it has more practical meaning for human disease prevention and control.

In this paper, based on the research of the human infectious diseases models, we study impulsive differential equation model on the spreading of plant diseases and insect pests under the condition of the total number of forest plants $N(\mathrm{t})$ is change with time. Considering vertical transmission(vertical transmission for plants is that the trees at the moment planted carry pests) and horizontal transmission, we spray insecticide for plants in fixed points $t=n T$.Assuming the standard incidence of plants disease $\beta(t)$ periodically change over time, the period is $T$, we set up impulsive spraying insecticide SIRS model with single species.

\section{THE SIRS MODEL WITH PULSE SPRAYING PESTICIDE}

The plants in the presence of diseases and insect pests area can be devided into three types: Susceptible( $\bar{S}$ ), Infections $(\bar{I})$ and Recovered $(\vec{R})$.we suppose that the total number of forest plants $N(\mathrm{t})$ is not a constant, $N(\mathrm{t})=\bar{S}(\mathrm{t})+\bar{I}(\mathrm{t})+\bar{R}(\mathrm{t}) \quad$,considering the standard incidence $\beta(t) \frac{S}{N} I$ which periodically change with time and the pulse pesticide spraying strategy ,we construct the following SIRS model:

$$
\left\{\begin{array}{l}
\frac{d \bar{S}}{d t}=b_{1}(\bar{S}+\bar{R})+b_{1} \rho \bar{I}-\frac{\beta(t)}{N(t)} \bar{S} \bar{I}+\delta \bar{R}-d_{1} \bar{S}, \\
\frac{d \bar{I}}{d t}=\frac{\beta(t)}{N(t)} \bar{S} \bar{I}-\left(d_{1}+d_{2}\right) \bar{I}+(1-\rho) b_{1} \bar{I}-\gamma \bar{I}, \\
\frac{d \bar{R}}{d t}=\gamma \bar{I}-d_{1} \bar{R}-\delta \bar{R}, \\
\bar{S}\left(t^{+}\right)=(1-p) \bar{S}(t), \\
\bar{I}\left(t^{+}\right)=(1-q) \bar{S}(t), \\
R\left(t^{+}\right)=\bar{R}(t)+p \bar{S}(t)+q \bar{I}(t) .
\end{array}\right\} t=n T
$$

Where $b_{1}$ is the coefficient of the trees planting. $d_{1}(N)$ is the natural cutting coefficient. $d_{2}$ is coefficient of dying from disease. $\gamma$ is recovery rate. $\frac{1}{\delta}$ is the average immune period. $\rho(0<\rho<1)$ is the proportionality coefficient of the newly planted trees being normal trees. $p, q$ respectively represent the proportion of susceptible(S)and infections(I) being succeed in spraying pesticide at $t=n T(\mathrm{n} \in \mathrm{Z}) \cdot \beta(t)$ is constant or given by 


$$
\beta(t+T)=\beta(t), \beta(t)=c_{0}\left(1+c_{1} \cos 2 \pi t\right)
$$

If $\beta(t)$ is a periodic function, $c_{0}$ is a constant, $0 \leq c_{1} \leq 1$ determines the amplitude of seasonal variation. $T$ is the time between two consecutive pulse vaccinations. $T \in R$, when $\beta(t)$ is constant. $T \in \mathrm{N}$, when $\beta(t)$ is a periodic function.

From the model (1),we obtain,

Where

$$
\frac{d N(t)}{d t}=\left(b_{1}-d_{1}(N)\right) N(t)-d_{2} \bar{I}
$$

(1) $d_{1}(N)=d$ is constant. When $d_{2}=0$,It exist exponential growth(when $b_{1}>d$ ), zero growth(when $b_{1}=d$ )or index decline (when $b_{1}<d$ ).

(2) $d_{1}(N)=d(N)$ non-decreasing and make $N(t)$ logistic growth to an equilibrium $N^{*}$. Of course, $d(N(t)) \geq d\left(N\left(t_{0}\right)\right)$.

\section{THE EXISTENCE AND LAS OF DISEASE-FREE} PERIODIC SOLUTION

By substituting $\frac{d N(t)}{d t}=\left(b_{1}-d_{1}(N)\right) N(t)-d_{2} \bar{I}$ to model (1) , we obtain:

$\left\{\begin{array}{l}\frac{d \bar{S}(t)}{d t}=\left(b_{1}+\delta\right) N(t)-\left(d_{1}+\delta\right) \bar{S}+\left(b_{1} \rho-b_{1}-\delta\right) \bar{I}-\frac{\beta(t)}{N(t)} \bar{S} \bar{I}, \\ \frac{d \bar{I}}{d t}=\frac{\beta(t)}{N(t)} \bar{S} \bar{I}-\left(d_{1}+d_{2}\right) \bar{I}+(1-\rho) b_{1} \bar{I}-\gamma \bar{I}, \\ \bar{S}\left(t^{+}\right)=(1-p) \bar{S}(t), \\ \bar{I}\left(t^{+}\right)=(1-q) \bar{I}(t) .\end{array}\right\} \quad \begin{aligned} & t=n T \\ & t \neq n T\end{aligned}$

We make $(\bar{S}(t), \bar{I}(t), \bar{R}(t))=N(t)(S(t), I(t), R(t))$, then

and

$$
\frac{d N(t)}{d t}=\left(b_{1}-d_{1}-d_{2} I\right) N(t)
$$$$
\left\{\begin{array}{l}
\frac{d S}{d t}=\left(b_{1}+\delta\right)(1-S)+\left(b_{1} \rho-b_{1}-\delta\right) I-\left(\beta(t)-d_{2}\right) S I, \\
\frac{d I}{d t}=\beta(t) S I-\left(d_{2}+\gamma+b_{1} \rho\right) I+d_{2} I^{2}, \\
S\left(t^{+}\right)=(1-p) S(t), \\
I\left(t^{+}\right)=(1-q) I(t) .
\end{array}\right\} \quad t=n T \quad t \neq n T
$$

This is similar to the literature, we can easily obtain one disease-free periodic solution $\left(S^{*}(t), 0\right)$ of system (3),

We can conclude that:

Theorem1 If the basic reproductive rate of plants $R(\mathrm{~T})<1$, i.e.

$$
\frac{p e^{-\left(b_{1}+\delta\right) T}\left(1-e^{-\left(b_{1}+\delta\right) T}\right)}{\left(b_{1}+\delta\right) T\left(1-p-e^{-\left(b_{1}+\delta\right) T}\right)}<\frac{m_{2}}{m_{1}},
$$

Where

$$
\begin{aligned}
& m_{1}=c_{0}\left(\mathrm{c}_{1} \alpha_{1}+\alpha_{1}^{2}+4 \pi^{2}\right), m_{2}=\left(\alpha_{3}-\mathrm{c}_{0}\right)\left(4 \pi^{2}+\alpha_{1}^{2}\right), \\
& \alpha_{1}=b_{1}+\delta, \alpha_{3}=d_{2}+\gamma+b_{1} \rho .
\end{aligned}
$$

The disease-free periodic solution $\left(S^{*}(t), 0, R^{*}(t)\right)$ is LAS.

IV. THE GAS OF DISEASE-FREE PERIODIC SOLUTION making $S(t)=\frac{\bar{S}(t)}{N(t)}$, thus the system(3) turns into:

$$
\left\{\begin{array}{l}
\frac{d S}{d t}=\left(b_{1}+\delta\right)(1-S)+\left(b_{1} \rho-b_{1}-\delta\right) \frac{\bar{I}(t)}{N(t)}-\left(\beta(t)-d_{2}\right) S \frac{\bar{I}(t)}{N(t)}, \\
\frac{d \bar{I}}{d t}=\beta(t) S(t) \bar{I}(t)-\left(d_{1}+d_{2}\right) \bar{I}(t)+(1-\rho) b_{1} \bar{I}-\gamma \bar{I}, \\
S\left(t^{+}\right)=(1-p) S(t), \\
\bar{I}\left(t^{+}\right)=(1-q) \bar{I}(t) .
\end{array}\right\} \quad t=n T
$$

Considering the biological significance of system (4),we have invariant set:

$$
\Omega=\{(S(t), \bar{I}(t)) \mid S(t) \in[0,1], \bar{I}(t) \leq 1-\bar{S}(t)\} .
$$

Theorem 2 If $\beta(\mathrm{t}) \geq d_{2}$ and the characteristic index of the following system (5) $\psi_{1}, \psi_{2}$ satisfy $\max \left\{\left|\psi_{1}\right|,\left|\psi_{2}\right|\right\}<1$

$\left\{\begin{array}{l}\frac{d Y}{d t}=\beta(t) S^{*}(t) Y-\left(\gamma+b_{1} \rho\right) Y, \quad t \neq n T \\ Y\left(t^{+}\right)=(1-q) Y(t), \quad t=n T \\ Y\left(t_{0}\right)=I_{0} .\end{array}\right.$

The system (3) is GAS in the set $\Omega$.

Proof: Considering the subsystem of (3),

$\left\{\begin{array}{l}\frac{d S}{d t}=\left(b_{1}+\delta\right)(1-S)+\left(b_{1} \rho-b_{1}-\delta\right) I-\left(\beta(t)-d_{2}\right) S I, \quad t \neq n T \\ S\left(t^{+}\right)=(1-p) S(t), \quad t=n T \\ S\left(t_{0}\right)=S_{0} .\end{array}\right.$

because $b_{1} \rho-b_{1}-\delta<0$, so when $\beta(t) \geq d_{2}$,

$\left(b_{1}+\delta\right)(1-S)+\left(b_{1} \rho-b_{1}-\delta\right) I-\left(\beta(t)-d_{2}\right) S I \leq\left(b_{1}+\delta\right)(1-S)$

Thus we have the following impulsive differential system:

$$
\left\{\begin{array}{l}
\frac{d X}{d t}=\left(b_{1}+\delta\right)(1-X), \quad t \neq n T \\
X\left(t^{+}\right)=(1-p) X(t), \quad t=n T \\
X\left(t_{0}\right)=S_{0} .
\end{array}\right.
$$

We can get the analytical solution of this system. According to the comparison theory of impulsive differential system, we can know it satisfy: 


$$
S(t) \leq X(t), X(t) \rightarrow S^{*}(t)(t \rightarrow+\infty) .
$$

From $0 \leq I \leq 1, S(t) \leq X(t)$, we know,

$$
\begin{aligned}
\beta(t) S I-\left(d_{2}+\gamma+b_{1} \rho\right) I+d_{2} I^{2}= & \beta(t) S I-\left(\gamma+b_{1} \rho\right) I+d_{2} I(I-1) \\
& \leq \beta(t) X I-\left(\gamma+b_{1} \rho\right) I
\end{aligned}
$$

Considering the system:

$$
\left\{\begin{array}{l}
\frac{d Y}{d t}=\beta(t) X Y-\left(\gamma+b_{1} \rho\right) Y, \quad t \neq n T \\
Y\left(t^{+}\right)=(1-q) Y(t), \quad t=n T \\
Y\left(t_{0}\right)=I_{0} .
\end{array}\right.
$$

We know that the system (8) has one equilibrium point $Y(t)=0$, and $I(t) \leq Y(t)$. For $X(t) \rightarrow S^{*}(t)(t \rightarrow+\infty)$, the system (10) and (6) have the same asymptotic property. If the condition of the theorem 2 is tenable, we obtain

$$
\begin{aligned}
\lim _{t \rightarrow \infty} I(\mathrm{t})= & 0 \cdot \\
& \begin{cases}\frac{d S}{d t}=\left(b_{1}+\delta\right)(1-S), & t \neq n T \\
S\left(t^{+}\right)=(1-p) S(t), \quad t=n T & t \\
S\left(t_{0}\right)=S_{0} . & \end{cases}
\end{aligned}
$$$$
\lim _{t \rightarrow \infty} Y(t)=0, \limsup _{t \rightarrow \infty} I(t) \leq \lim _{t \rightarrow \infty} \sup Y(t)=0 \text {. For } I(\mathrm{t}) \geq 0,
$$

Therefore $S(t) \rightarrow S^{*}(t)(t \rightarrow+\infty)$, Further more,

$$
(S(t), I(t)) \rightarrow\left(S^{*}(t), 0\right)(t \rightarrow+\infty),
$$

i.e., the pest-eradication periodic solutions is GAS. addition,

$b_{1}=d_{1}(N)$, since $\frac{d N(t)}{d t}=\left(b_{1}-d_{1}(N)\right) N(t)-d_{2} \bar{I}=-d_{2} \bar{I}$,we get:

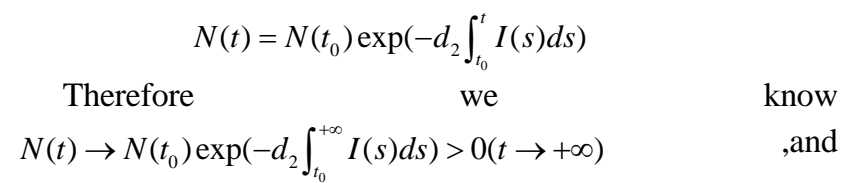
$\bar{I}(t)=I(t) N(t), \bar{I}(t) \rightarrow 0$,i.e., the total eradication of pests is guaranteed. however if $b_{1}>d$ or $d_{1}(N)=d(N)$,i.e., the growth of plants is exponential or Logistic, the total eradication of pests can not be guaranteed. For these two cases, as follows, we give the conditions of GAS for the periodically total eradication solutions.

Theorem 3 If $\beta(t) \geq d_{2}$ and one of the following conditions is founding:

(1) the growth of forest plants is exponential and $d_{2} \geq 0$,

(2) the growth of forest plants is Logistic and $d_{2}>0$,

(3) the conditions of the GAS for the periodic solution of pests being totally eradicated in $\Omega$ is that the characteristic index $\omega_{1}, \omega_{2}$ of the following system (11) satisfy :

$$
\begin{gathered}
\quad \max \left\{\left|\omega_{1}\right|,\left|\omega_{2}\right|\right\}<1 \\
\left\{\begin{array}{l}
\frac{d Z}{d t}=\beta(t) S^{*} Z-\left(d\left(N_{0}\right)+d_{2}\right) Z+(1-\rho) b_{1} Z-\gamma Z, t \neq n T \\
Z\left(t^{+}\right)=(1-q) Z(t), \quad t=n T \\
Z\left(t_{0}\right)=I_{0} .
\end{array}\right.
\end{gathered}
$$

Where $N\left(t_{0}\right)=N_{0}$, if condition (1) is founding, $N\left(t_{0}\right)=d$.if condition (2)

is founding, $N\left(t_{0}\right)=N_{0}$.

Proof: since $N(t) \geq N\left(t_{0}\right)$,we gain:

$$
\begin{aligned}
\frac{d \bar{I}}{d t} & =\frac{\beta(t)}{N(t)} \bar{S} \bar{I}-\left(d(N)+d_{2}\right) \bar{I}+(1-\rho) b_{1} \bar{I}-\gamma \bar{I} \\
& =\beta(t) S \bar{I}-\left(d(N)+d_{2}\right) \bar{I}+(1-\rho) b_{1} \bar{I}-\gamma \bar{I} \\
& \leq \beta(t) S \bar{I}-\left(d\left(N\left(t_{0}\right)\right)+d_{2}\right) \bar{I}+(1-\rho) b_{1} \bar{I}-\gamma \bar{I}
\end{aligned}
$$

If inequation (10) is tenable, similar to the proof of theorem 2, we obtain $\quad \bar{I}(t) \rightarrow 0(t \rightarrow+\infty) \quad$,therefore, $\frac{d N(t)}{d t}=\left(b_{1}-d_{1}(N)\right) N(t)-d_{2} \bar{I} \quad$ is asymptotically stable and $N(t) \rightarrow+\infty(t \rightarrow+\infty)$ or $N(t) \rightarrow N^{*}(t \rightarrow+\infty)$, thus

$$
\begin{gathered}
\left(b_{1} \rho-b_{1}-\delta\right) \frac{\bar{I}(t)}{N(t)}-\left(\beta(t)-d_{2}\right) S \frac{\bar{I}(t)}{N(t)} \rightarrow 0(t \rightarrow+\infty) \\
\text { so } \frac{d S}{d t} \rightarrow\left(b_{1}+\delta\right)(1-S)(t \rightarrow+\infty) \text {, and } S(t) \rightarrow S^{*}(t)(t \rightarrow+\infty)
\end{gathered}
$$

\section{TWO EXAMPLES}

A.

We make

$$
\begin{aligned}
& \alpha_{1}=0.8, \alpha_{3}=0.12, c_{0}=0.07, c_{1}=0.05, b_{1}=\delta=0.4, \\
& \gamma=d_{2}=0.02, \rho=0.2, p=0.3, q=0.7, T=1
\end{aligned}
$$

By substituting above coefficient to model (3),we obtain:

$$
\left\{\begin{array}{l}
\frac{d S}{d t}=0.8(1-S)-0.72 I-[0.07(1+0.05 \cos 2 \pi t)-0.02] S I, \\
\frac{d I}{d t}=0.07(1+0.05 \cos 2 \pi t) S I-0.12 I+0.02 I^{2}, \\
S\left(t^{+}\right)=0.7 S(t), \\
I\left(t^{+}\right)=0.6 I(t) .
\end{array}\right\} t=n
$$

Solving ,we gain $m_{1}=3, m_{2}=2$,therefore $R(T)=0.5625<1$ and it satisfy the conditions of theorem 1.Then we simulate solution curve of model(3): 


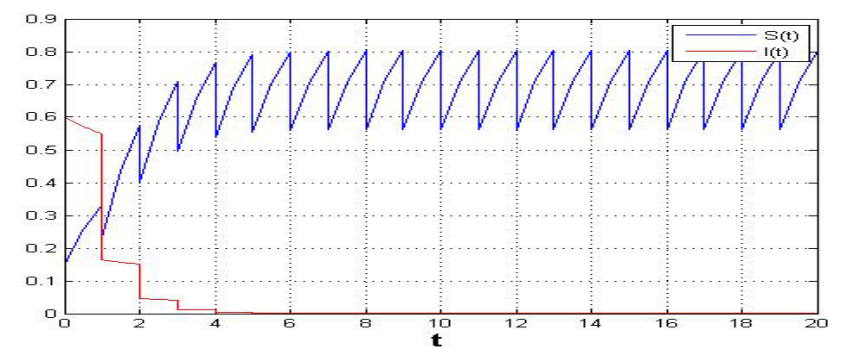

FIGURE I. THE SOLUTION CURVE OF $S(t), I(t)$.

As you can see in the above figures, the system has one disease-free periodic solution $\left(S^{*}(t), 0\right)$, and it is LAS.

B.

We make

$\alpha_{1}=0.4, \alpha_{3}=0.707, c_{0}=0.07, c_{1}=0.1, b_{1}=0.25, \delta=0.15$,

$\gamma=0.35, d_{2}=0.3, \rho=0.2, p=0.2, q=0.4$

By substituting above coefficient to model (3),we gain:

$$
m_{1}=16, m_{2}=12,
$$

therefore $R(T)=1.13>1$, Then we simulate solution curve of model(3):

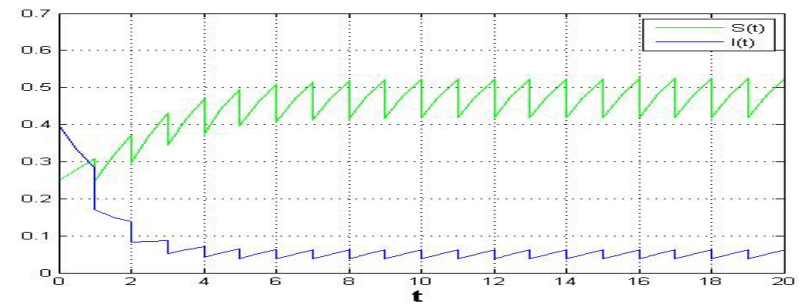

FIGURE II. THE SOLUTION CURVE OF $S(t), I(t)$.

As you can see in the above figures, the system has one disease-free periodic solution $\left(S^{*}(t), 0\right)$ and $S(t) \rightarrow S^{*}(t)(t \rightarrow+\infty)$, however $I(t)$ does not go to zero asymptotically. So the theorem 1 is correct.

We can use the same method to testify theorem 2 and theorem 3 and I will not repeat them now.

\section{ACKNOWLEDGEMENTS}

This work is supported by the National Science Foundation of China (61273016) and National Science Foundation of Zhejiang (Y6100611).

\section{REFERENCES}

[1] Z.H.Lu, X. B.Chi, L. S. Chen, The effect of constant and pulse vaccination on SIR epidemic model with horizontal and vertical transmission[J]. Mathematical and Computer Modelling, 2002,36(910):1039-1057.

[2] Ganna Rozhnova, Ana Nunes, SIRS Dynamics on Random Networks:Simulations and Analytical Models[J].ICST Institute for Computer Scinces,Social Informatics and Telecommunications Engineering,2009,1(4):792-797

[3] Y.Y.Zhang, D.J.Wang, Stability of an age structured forest insect pests model with vertical transmission and continuum vaccination[J]. Journal of Biomathematics,2012, 27(3):489-493.
[4] J.L. Ma, David J.D.Earn.Generality of the Final Size Formular for an Epidemic of a Newly Invading Infectious Disease[J].Bulletin of Mathematical Biology,2006,68:679-702.

[5] Jean M.Tchuenche, Alexander Nwagwo. Local stability of an SIR Epidemic Model and Effect of Time Delay[J].Mathematical Methods in the Applied Sciences, 2009,32:2160-2175.

[6] Gao Bei-bei,WANG Ding-jiang. Periodic Solution of the Plants Diseases Model with Impulsive Spraying Insecticide[J]. Journal of Biomathematics,2014.

[7] D’Onofrio A. Stability properties of pulse vaccination strategy in SEIR epidemic model[J].Mathematical Biosciences,2002,179(1):5772.

[8] T.Y.Wang, C.H Chen, L. S. Chen, Periodic Solution of a Microbial Pesticide Model with the Monod Growth Rate and Impulsive Feedback control[J]. Journal of Biomathematics,2013,28(4):577-585.

[9] C.J.Wei, L. S. Chen, A Leslie-Gower pest management model with impulsive state feedback control[J]. Journal of Biomathematics, 2012, 27(4): 621-628.

[10] X.B.Zhang,H.F.Huo,X.K.Sun, Q.Fu , The differential susceptibility SIR epidemic model with time delay and pulse vaccination[J]. Korean Society for Computational and Applied Mathematics, 2009,

[11] B. Shulgin, L. Stone, Z. Agur, Theoretical examination of pulse vaccination policy in the SIR epidemic model[J],Math. Comp. Model. 31(4/5) (2000) 207.

[12] B.B.Gao,D.J.Wang, Periodic Solution of the Plants Diseases Model with Impulsive Spraying Insecticide[J]. Acta Analysis Functionalis Applicata, 2014.

[13] X.Y.Song, H.J.Guo, X.Y.Shi, Theory and Application of Impulsive Differential Equations [M].Beijing: Science Press, 2008. 